\title{
Development of Analysis Method using GEANT4 for Cosmic Ray Radiography
}

\section{Mitsuaki Kuno*}

Physics of science, Nagoya University, Furo-cho, Chikusa, Nagoya, Aichi, Japan

E-mail: kunoeflab.phys.nagoya-u.ac.jp

\section{Kunihiro Morishima}

Nagoya University

\section{Akira Nishio}

Nagoya University

\section{Yuta Manabe}

Nagoya University

\section{Nobuko Kitagawa}

Nagoya University

\begin{abstract}
Cosmic ray radiography is the inner investigation technology for large structures using by the muon. The "Scan Pyramids Mission" was launched in September of 2015 for observation of Egyptian Pyramid. We had installed nuclear emulsion detectors in the Bent Pyramid and the Khufu's Pyramid. Cosmic ray radiography simulator was developed using by GEANT4 to estimate expected image by the survey. By the comparison between experiment data at the Descending Corridor in the Khufu's Pyramid and simulation result, the unknown void had been discovered behind the entrance of the Khufu's Pyramid.
\end{abstract}

35th International Cosmic Ray Conference - ICRC2017

12-20 July, 2017

Bexco, Busan, Korea

${ }^{*}$ Speaker. 


\section{Introdaction}

Cosmic ray radiography is non destructive examination technology for large structures. The density length (= material density $\times$ path length) are estimated to compare with number of cosmic ray muons on the ground and detected muons that path through structures. if we knew the structure size, we can calculated its density distribution as like X-ray radiography. In 1969, this technic for the first time was used to search for hidden chamber in the Khafre's Pyramid at Egypt by L.W. Alvarez, however new chamber was not appeared [1]. In September of 2015, the "Scan Pyramids Mission" was started for scientifically investigating Egyptian pyramids with ministry of archaeology at Egypt and international collaboration. Red, Bent, Khafre's and Khufu's pyramids are observation targets. We have installed nuclear emulsion detectors in the Bent Pyramid in December of 2015 and the Khufu's pyramid in June of 2016.

Nuclear emulsion films can be seen to detect charged particle tracks that were lined up silver grain when these were grown up until visible with a optical microscope by chemical development. Films have been used neutrino oscillation experiment (OPERA [2]) and gamma ray telescope (GRAINE [3]). The muon track can be visible a straight line (Figure 1a). The image are made by to count number of muon tracks and to decide angles of incoming muons in the film. The radiography is given high image quality by angle resolution of sub-mrad of the emulsion film. The nuclear emulsion detector using by radiography is $25 \mathrm{~cm} \times 35 \mathrm{~cm}$ and thickness about $300 \mu \mathrm{m}$. Films can be installed anywhere because it need not power supply and is a compact device (Figure 1b).

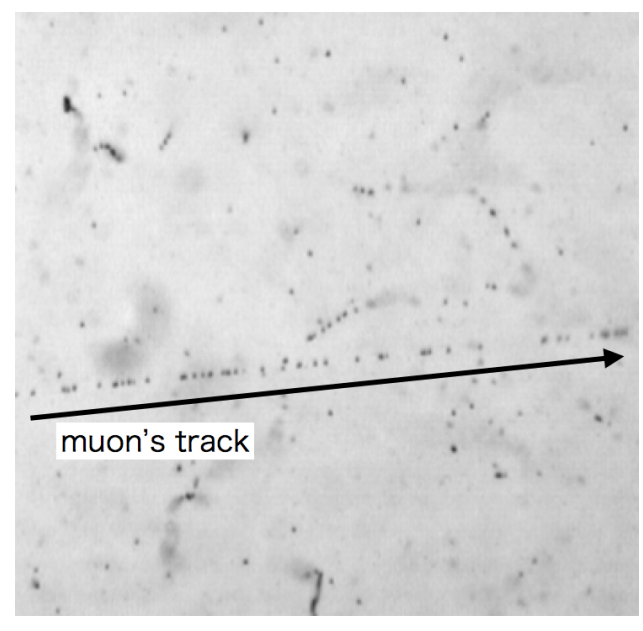

(a) Microscopic image in the developed film

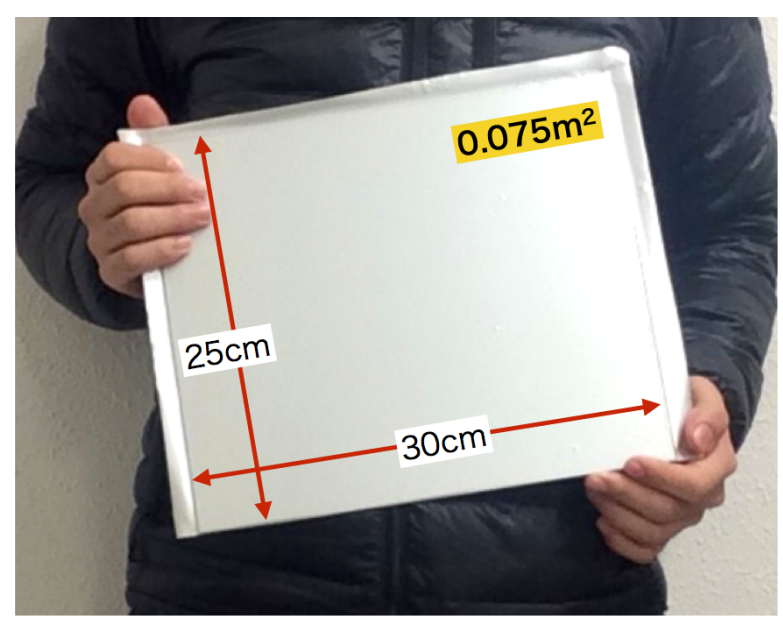

(b) The nuclear emulsion detector

Figure 1: The nuclear emulsion for cosmic ray radiography 


\section{Cosmic ray radiography simulator}

Cosmic ray radiography simulator will be need to include next two things. First of all, cosmic ray muons rain any angles and muon energy distribution have wide range. The muon spectral equation on the ground $I_{0}$ are given by S. Miyake [4]

$$
I_{0}=\frac{174}{5 E \cos \theta+400}\left(5 E+\frac{10}{\cos \theta}\right)^{-1.57} \frac{E+15}{E+10+5 / \cos \theta} \quad\left[\mathrm{cm}^{-2} \mathrm{~s}^{-1} \mathrm{sr}^{-1}\right]
$$

where $E$ is muon energy that required above $1 \mathrm{GeV}$ and $\theta$ is zenith angle.

GEANT4 that is able to calculate physics process is Monte Carlo simulation [5]. The particle generator code of GEANT4 for cosmic ray radiography was developed by including muon flux equation (2.1). Figure 2 is compare to between generated muons at the simulation and the muon flux formula. The simulation was run at $2 \times 10^{9}$ muons.

(a)

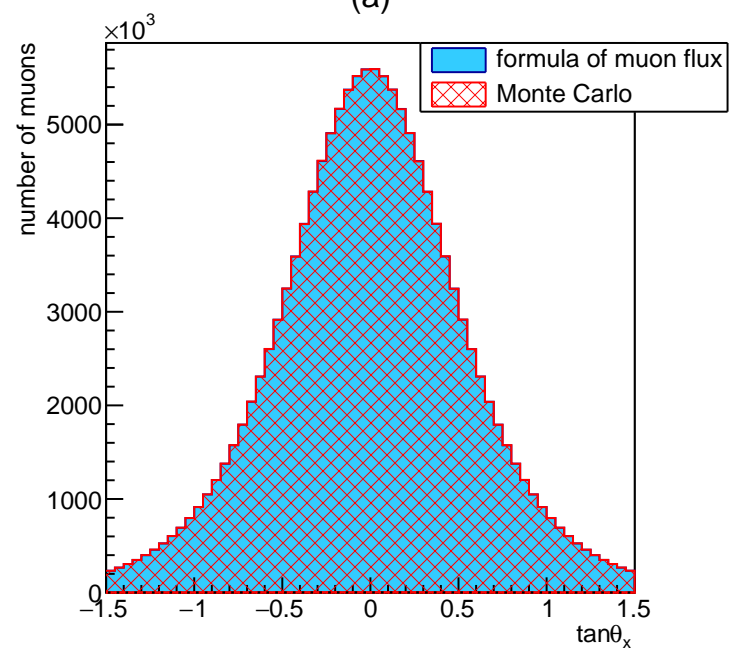

(b)

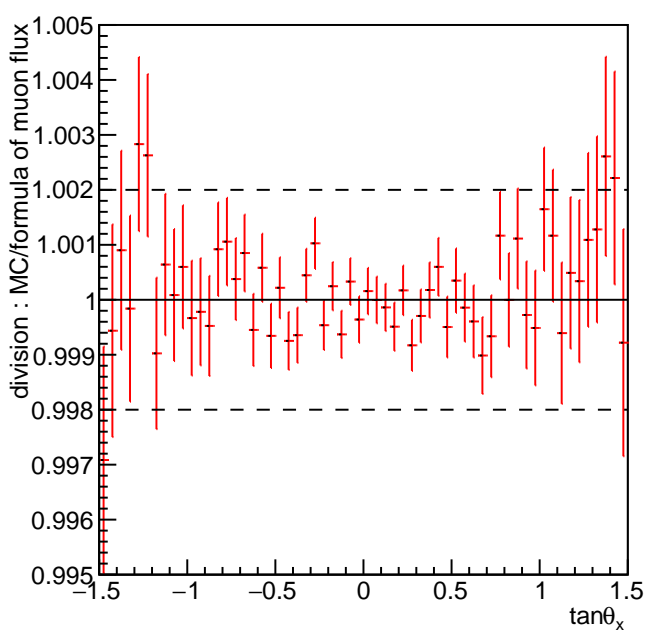

Figure 2: The generated angle distribution of muon spectral in the simulation

Figure 2a is projection of number of muons of equation (2.1) and the simulation that have been normalized. Figure $2 b$ is simulation divided by muon flux equation (2.1) and statistical error is $1 \sigma$. These $\mathrm{x}$ axis is zenith angle of arrived muons to the detector and is written at $\tan \theta$. The divided value is smaller than $\pm 0.2 \%$ and is enough for the statistical fluctuation of radiography imaging. Second of all, radiography imaging resolution would be influenced by multiple coulomb scattering in materials. In addition, the resolution are deteriorated when detectors surface size are not enough smaller than the observation object. The arrived point of exposure muons in the simulation is moved by the scattering effect in the structure. The exposer area of muons would be written by

$$
S=\left(d l_{x}+2 d_{s}\right) \times\left(d l_{y}+2 d_{s}\right)
$$

where $d l_{x}$ and $d l_{y}$ are one side of the detector and $d_{s}$ is the expanse of muons by the scattering effect from the center of detector. The simulation need to be run that considered exposer area. 
The muon flux $I$ when number of detected muons $N$ is obtained by

$$
I=\frac{N / \cos ^{4} \theta}{A \times T \times d s r} \quad\left[\mathrm{~cm}^{-2} \mathrm{~s}^{-1} \mathrm{sr}^{-1}\right]
$$

where $\theta$ is incoming direction to the detector of muons, $A$ is the surface area of detector, $T$ is the observation period and $d s r$ is 1 pixel size of image. The minimum energy that is able to arrive to detectors though structures is estimated to compare with muon flux on the ground.

$$
I=I_{0}^{\prime}\left(E^{\prime}, \theta\right)
$$

where $I$ is detected muon flux, $I_{0}^{\prime}$ is muon flux on the ground when energy threshold is $E^{\prime}$ and one direction of arrived muons is $\theta$. Thus $E^{\prime}$ would be minimum energy that can be incoming the detector though the object. The density length can be calculated by mean of energy loss $d E / d x$ in the material and minimum energy $E^{\prime}$.

$$
\text { density length }=\frac{E^{\prime}}{d E / d x} \quad\left[\mathrm{~g} \mathrm{~cm}^{-2}\right]
$$

For instance, the prospective distance of room is estimated by multiplication of the density length and expected structure density. Cosmic ray radiography can be discovered the anomaly by to compare with muon flux and the density length of experiment data and simulation result.

\section{The Bent Pyramid}

The Bent Pyramid was built by Sneferu in Dahsur of Egypt. First of all, we have launched pyramid survey at the Bent Pyramid. The purpose is to confirm that cosmic ray radiography can carry out in the pyramid.
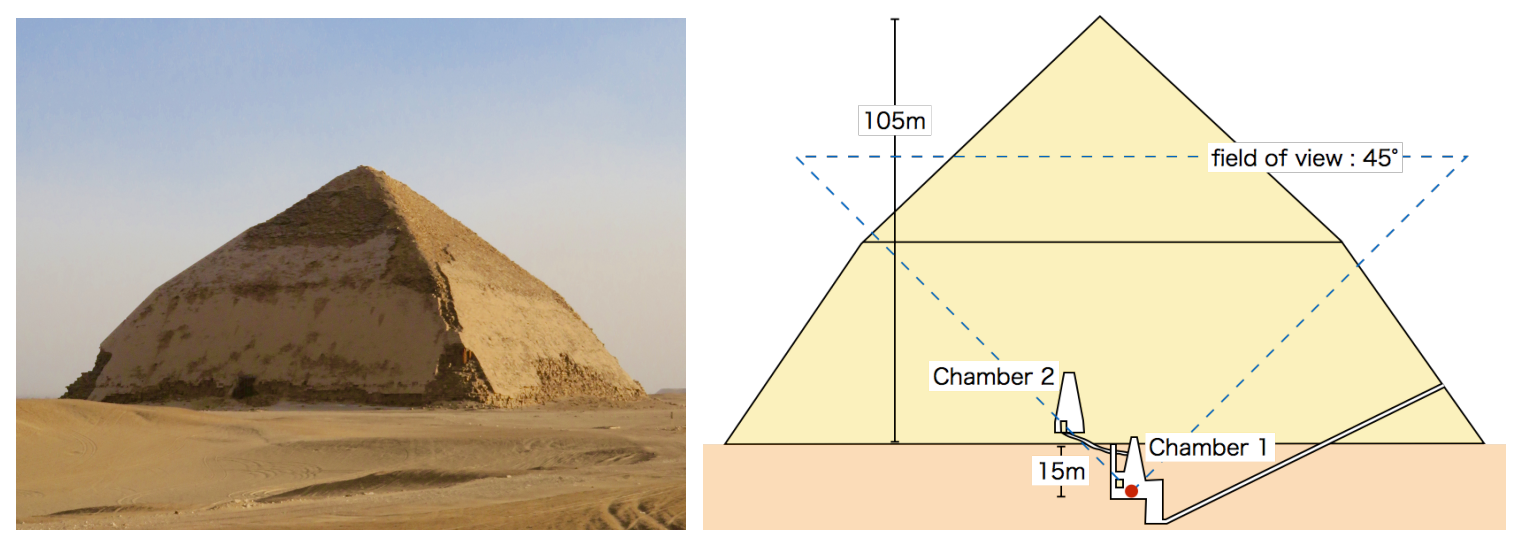

Figure 3: The Bent Pyramid

In December of 2015, we had installed emulsion detectors of $3 \mathrm{~m}^{2}$ to Chamber 1 in the Bent Pyramid (Figure 3) and the observation period was 40 days. The filed of view of detector is 45 degrees $(\tan \theta=1)$ and can be seen Chamber 2 from Chamber 1 . Cosmic ray radiography simulator 
with GEANT4 is able to estimate expected image at any detector position. The simulation for Bent Pyramid survey have been calculated and is statics of 600 days. The left of Figure 4 is the radiography image of muon flux with simulation result. The $\mathrm{x}$ and $\mathrm{y}$ axis is direction of arrived muons to detector that is $\tan \theta$. The Chamber 2 would to be seen position as like the image. The right of Figure 4 is muon flux of experiment result from the Chamber 1 . The chamber 2 in the observation result would be able to detect to same point with simulation. We have detected the known chamber in the pyramid for the first time in the world using by cosmic ray radiography.
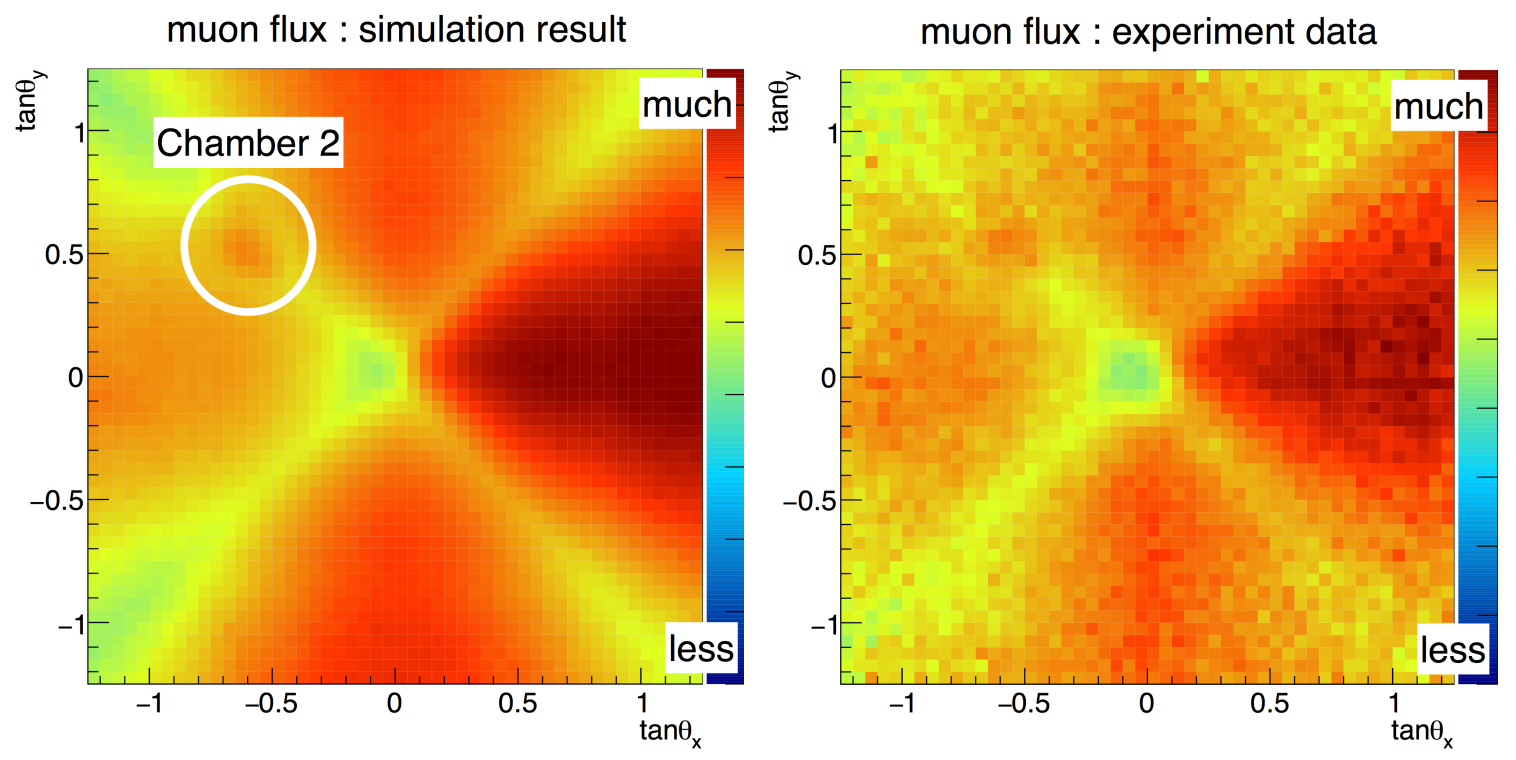

Figure 4: The radiography image of estimated by the simulation and experiment result at the Bent Pyramid

\section{The Khufu's Pyramid}

The Khufu's Pyramid was constructed by Khufu in Giza of Egypt that is the most largest pyramid around the world. This pyramid has a lot of hypothesis about unknown chambers. The investigation of Khufu's Pyramid was approved by the result of the Bent Pyramid study with nuclear emulsion detectors. In June of 2016, we have started observation in the Khufu's Pyramid. The Descending Corridor (Figure 5) is a small path of $1 \mathrm{~m}$ by $1.2 \mathrm{~m}$ that continue from pyramid entrance to an underground room and has slope of 26 degrees. The nuclear emulsion film that is a compact detector can be installed such as a narrow passage. 

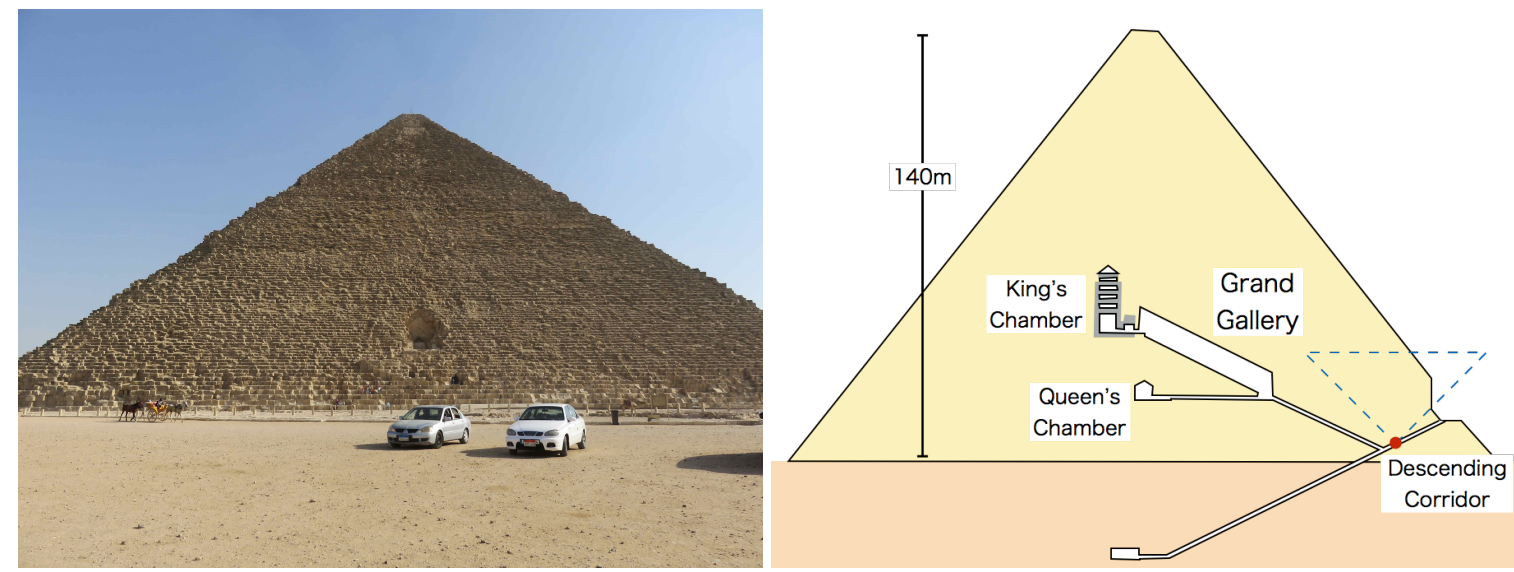

Figure 5: The Khufu's Pyramid
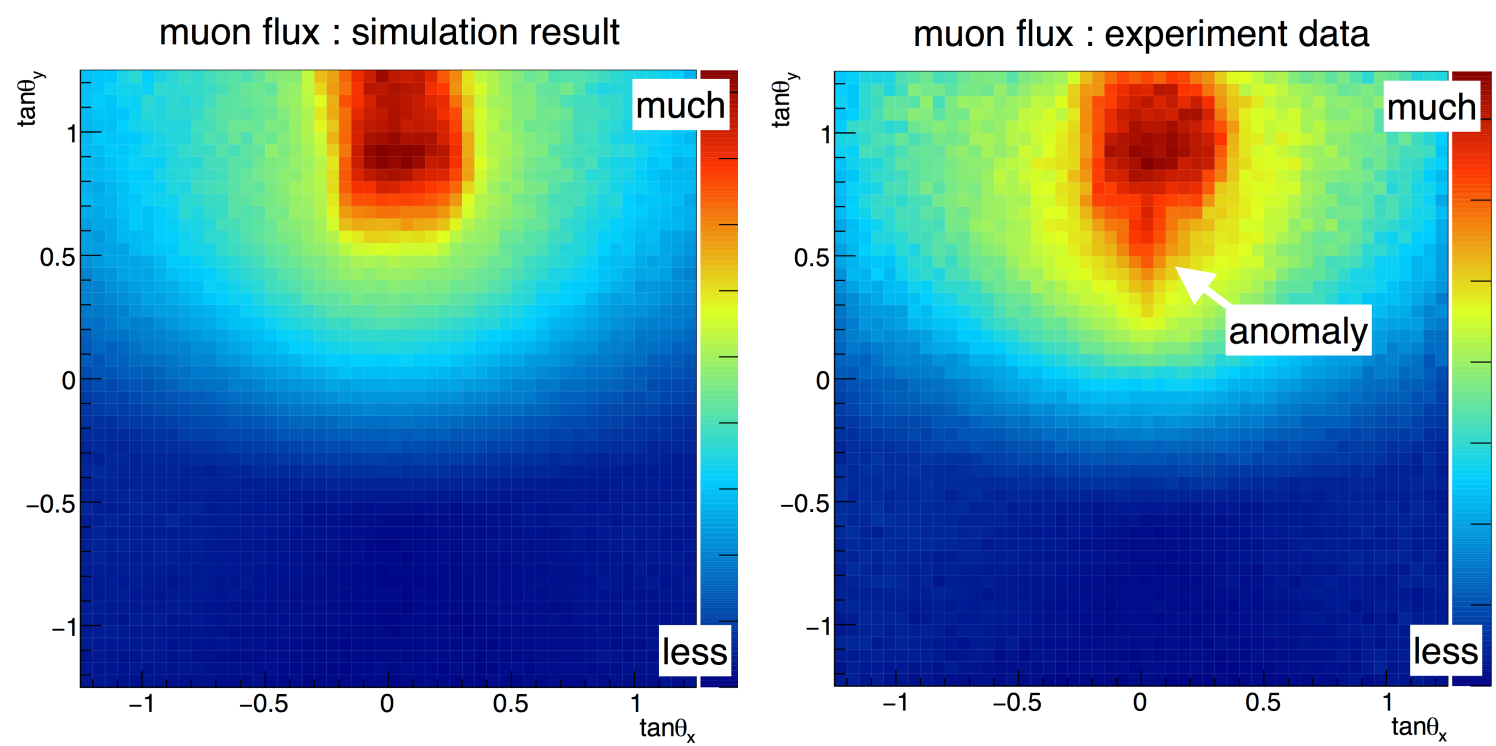

Figure 6: The radiography image of estimated by the simulation and experiment result at the Descending Corridor of the Khufu's Pyramid

We have studied at the Descending Corridor and the observation of 67 days have been started by emulsion detectors. The left of Figure 6 is a simulated radiography image using by one detector's area of $0.075 \mathrm{~m}^{2}$ and statistic are 150 days. The image is zenith angles $(\tan \theta)$ of detected muons. The right of Figure 6 is experiment result in 67 days for a detector of $0.075 \mathrm{~m}^{2}$. The observation data have been gained more muons than expected at specific angle area. The estimated muon excess is more than $5 \sigma$. This depth of anomaly is calculated be at $1.8 \pm 0.3 \mathrm{~m}$ when the Lime Stone density are $2.2 \mathrm{~g} \mathrm{~cm}^{-3}$. It suggests the existence of unknown void behind the entrance of the Khufu's Pyramid. This result are investigated more detail by installed some films at variety position of the Descending Corridor. 


\section{Conclusion}

The emulsion film had been installed in the Bent Pyramid and the Khufu's Pyramid for investigation of pyramids by the cosmic ray radiography. The simulator was developed by reproducing formula of muon flux in the particle generator code of GEANT4 and can estimated that expected image. The unknown void was discovered by comparison between experiment data at the Descending Corridor in the Khufu's Pyramid and simulation.

\section{Acknowledgement}

The "Scan Pyramids Mission" was inaugurated by Egyptian ministry of archaeology and HIP (Heritage Innovation Preservation Institute in France) in September of 2015. The authors thank staffs of the project and the GEM-CC (The Grand Egyptian Museum Conservation Center).

\section{References}

[1] Luis W. Alvarez et al, Search for Hidden Chambers in the Pyramids, Science 167 (3919) p. 832-839.

[2] R. Acquafredda et al, First events from the CNGS neutrino beam detected in the OPERA experiment, New J. Phys. 8 (303) [hep-th/2001033].

[3] S. Takahashi et al, GRAINE 2015, a balloon-borne emulsion $\gamma$-ray telescope experiment in Australia, PTEP 2016 (7) 073F01.

[4] S. Miyake, Denver, in proceedings of 13th International Cosmic Ray Conference 5 (1973) p. 3638.

[5] S.Agostinelli et al, Geant4 - a simulation toolkit, PHYS RES. A506 (3) p. 250-303. 